\title{
Functional decline two weeks before hospitalization in an elderly population
}

\author{
Gianluca Isaia, Mario Bo, Nicoletta Aimonino, Giovanni Carlo Isaia, Giuliana Michelis, \\ Cristina Miceli, Luisa Balcet, Alessandra Tizzani and Mauro Zanocchi \\ Geriatric Section, Department of Medicine and Surgery, University of Torino, Torino, Italy
}

\begin{abstract}
Introduction: The use of hospital care increases significantly with age. Older people coming to the emergency department have three to seven times higher rates of hospitalization than younger people. Hospitalization can lead to loss of independence and also need for long-term care at discharge. Methods: A prospective observational non-randomized study of elderly patients admitted to the Department of Geriatric Medicine, University of Torino, Italy, between October 2006 and May 2007. Results: The mean age of 123 subjects was $82.2 \pm 7.3$ years. Among patients with dependence two weeks before admission, as reported by their caregivers, bathing, toileting and dressing were the most compromised $A D L$ functions. In the same patients, shopping and transportation were reported as the most frequent IADL functions lost. Functional decline before hospitalization was significantly associated with a worsening in functional decline during the length of stay ( $R R$ 2.2, CI 1.05-4.66) and with discharge to a long-term facility (RR 2.1, CI 1.04-4.44). Conclusions: As functional decline two weeks before hospitalization influences hospital discharge and functional outcomes, it is certainly interesting to evaluate the impact of a dedicated early rehabilitation program for elderly patients. A well-structured program involving geriatricians, physiatricians and physiotherapists could be helpful for better short-term prognoses.

(Aging Clin Exp Res 2010; 22: 352-355)

o2010, Editrice Kurtis
\end{abstract}

\section{INTRODUCTION}

Mean age is increasing everywhere, especially in developed countries, and this determines a higher need for hospital care. Among old persons, the use of hospital care increases significantly with age (1). Older people coming to the emergency department have three to seven times higher rates of hospitalization than younger people $(2,3)$.

Low mobility and bed rest commonly occur during hospitalization and may increase functional impairment (4). Palmer et al. (5) demonstrated that hospitalization in the elderly can lead to loss of independence, especially in cases of long in-hospital stays. Disability, especially for the oldest old, leads to restrictions in the ability to perform normal activities of daily living (6).

This study aimed at better understanding of the functional changes in elderly people requiring hospital admission for acute illnesses. Patients may modify these activities during hospitalization, but these changes may manifest even some days before hospitalization, and functional impairment is sometimes one of the causes for hospital admission.

\section{MATERIALS AND METHODS}

A prospective observational non-randomized study of elderly patients admitted to the Department of Geriatric Medicine, University of Torino, Italy, between October 2006 and May 2007.

Within 24 hours of hospital admission, patients were clinically and functionally assessed and data on age, sociodemographic and living conditions were collected. Evaluation of functional status was performed by the Activities of Daily Living (ADL) (7) and Instrumental Activities of Daily Living (IADL) (8) scales two weeks before admission, at admission and at discharge. Caregivers were asked to list in which items of the ADL and IADL scales their patients were impaired two weeks before hospitalization.

The time-point of two weeks was selected because it is far enough in the past to reflect the patient's functional status in general, before the onset of the acute illness episode that required hospital admission, but recent enough for respondents to be able to recall the patient's functional status reliably (9).

Key words: Elderly, functional decline, hospitalization.

Correspondence: Gianluca Isaia, MD, Medical and Surgical Department, Geriatric Section, University of Torino, C.so Bramante 88, 10126 Torino, Italy.

E-mail: gianlucaisaia@yahoo.it

Received March 10, 2009; accepted in revised form September 30, 2009. 
The Acute Physiology and Chronic Health Evaluation II (APACHE II) (10) classification system, applied on admission, was used to identify the influence of acute disease on functional status, the Mini Mental State Examination (MMSE) (11) was used to assess cognitive status, and the Geriatric Depression Scale (GDS) (12) to assess mood status. Patients were considered depressed if their GDS was $>11$.

Information on the main causes of hospitalization, $\mathrm{co}^{-}$ morbidities and details of discharge were also collected.

Exclusion criteria were: patients transferred from other wards or awaiting surgery; patients from nursing homes; patients who were being considered for palliative care; patients who had already been discharged from hospital within 48 hours previously, or who were not discharged from the emergency department within 24 hours; patients without personal caregivers.

\section{Statistical analysis}

Data were compiled by one of the medical researchers with at least 5 years experience in collecting data and making functional evaluations of elderly people.

Student's $t$-test was used to study continuous variables and the $\chi^{2}$ test to evaluate non-parametric data. Variables significantly associated with functional decline, after univariate analysis, entered the multivariate analysis with logistic regression, and the stepwise-forward method was used to determine the contribution of significant results. For relative risks, the confidence interval is reported.

Data were analysed with SPSS 11.1 for Windows.

\section{RESULTS}

Two hundred and seventy-four patients were consecutively admitted to the Geriatric Ward of the San Giovanni Battista Hospital of Torino: 123 fulfilled the in-

Table 1 - Demographic and clinical baseline characteristics.

\begin{tabular}{lcc}
\hline & $\mathbf{n}(\mathbf{\%})$ & Mean \pm SD \\
\hline Age & & $82.2 \pm 7.3$ \\
Length of stay & $14.3 \pm 17.5$ \\
Female & $82(66.7)$ & \\
Male & $41(33)$ & \\
Living alone & $47(38.2)$ & $2.2 \pm 2$ \\
ADL & & $2.5 \pm 1.2$ \\
IADL & & $8.3 \pm 2.6$ \\
APACHE II & $23.9 \pm 8.1$ \\
MMSE & $5.6 \pm 4.1$ \\
GDS & \\
\hline ADL: Activities of Daily Living; APACHE II: Acute Physiology and Chronic \\
Health Evaluation; MMSE: Mini Mental State Examination; GDS: Geriatric \\
Depression Scale.
\end{tabular}

Table 2 - Number of ADL functions lost before hospitalization, during hospital stay and after discharge for whole cohort of 123 patients.

\begin{tabular}{llc}
\hline ADL functions lost & $\boldsymbol{p}$ \\
\hline Two weeks before vs Admission & $1.10 \pm 1.60$ vs $2.20 \pm 2.02$ & $<0.001$ \\
Two weeks before vs Discharge & $1.10 \pm 1.60$ vs $2.61 \pm 1.98$ & $<0.001$ \\
Admission vs Discharge & $2.20 \pm 2.02$ vs $2.61 \pm 1.98$ & ns \\
\hline
\end{tabular}

clusion criteria and were considered eligible. Fourteen patients $(5.1 \%)$ died during hospitalization. Forty-four (16\%) refused to participate in the study. Twenty-three $(8.4 \%)$ were awaiting surgery. Twenty-one $(7.4 \%)$ arrived from a nursing home. Thirty-two $(11.7 \%)$ were admitted for palliative care. Two $(0.7 \%)$ patients were not discharged within 24 hours from the Emergency Department and 15 (5.5\%) were transferred from another ward.

The mean age of the total sample was $82.2 \pm 7.3$ years (range 66-99). Demographic and baseline clinical characteristics are listed in Table 1.

Main diagnoses on admission were: hypertension (27.3\%), pain (21.2\%), bronchopneumonia (18.2\%), syncope (16.2\%), fever (6.1\%) and heart failure (6.1\%).

Main diagnoses on discharge were: cardiovascular diseases (34.4\%), cerebrovascular diseases $(8.3 \%)$, pneumonia (28.1\%), neoplasias (11.5\%), metabolic diseases (7.3\%) and dementia (7.3\%).

Functional impairment two weeks before hospitalization was manifest in half the sample evaluated (50.4\%). Two weeks before hospitalization, lost ADL functions were significantly lower than on admission $(1.10 \pm 1.6$ vs $2.20 \pm 2.02 ; p<0.001)$ and functional impairment worsened during hospitalization (Table 2).

In patients with dependence two weeks before admission, bathing, toileting and dressing were the most compromised ADL functions (respectively 79\%, 66.1\% and $51.6 \%)$. Among the same patients, shopping and transportation were reported as the most frequent IADL functions lost (respectively 95.2\% and 83.9\%).

Univariate analysis showed that functional decline two weeks before hospitalization was related to discharge to a long-term facility (RR 3.05; CI 1.32-7.05) and to the functional decline detected during hospitalization (RR 3.36; CI 1.36-3.28).

Multivariate analysis showed that functional decline before hospitalization was significantly associated with worsening of the functional decline which occurred during the hospital stay (RR 2.2, CI 1.05-4.66) and with discharge to a long-term facility (RR 2.1, CI 1.04-4.44).

\section{DISCUSSION}

Functional decline in the elderly has been reported as a possible predictor for death or institutionalization 
(13). In addition, worsening of functional status during the two weeks before hospital admission may influence in-hospital functional decline. Before hospitalization, impairment of functional status may be due to factors not linked to length of hospital stay. In the elderly, a functional decline often occurs a few days before hospitalization, due to the onset of an acute disease or worsening of chronic illnesses, and the new condition of functional impairment may be the reason for hospital admission.

Patients who lose one or more ADL functions two weeks before admission probably have a higher risk of functional decline during hospitalization.

During hospitalization, specific factors may lead to functional impairment: patients may have problems in getting used to new surroundings or being alone without relatives for most of the hours during the day; they are often bedridden, due to acute illnesses.

Functional decline in hospitalized older people may occur frequently before admission. Hirsch et al. (14) reported that functional changes in hospitalized older people represent a complex process which may involve decline before admission. Sager et al. (15) reported that pre-admission disabilities are one of the most significant predictors of functional decline like age, gender, lower mental status and re-hospitalization.

An understanding of the rates, time-courses, and predictors of functional recovery in older adults hospitalized for medical illnesses is essential in planning for the needs of these patients and optimizing preventive and rehabilitative strategies for them.

One study (9), going back to 40 years ago, had already reported that functional status before the onset of acute illness may be an important survival predictor at 1 year follow-up. The importance of pre-hospital functional evaluation is a stronger predictor than assessment on admission.

The results of the present study, although they do not report survival outcomes, confirm the predictive effect of reported $\mathrm{ADL}$ status on worsening of functional decline during hospital stays.

Patients with functional decline two weeks before hospital admission also have a higher risk of being transferred to a long-term facility. This result is probably linked to the lower length of stay of pre-hospital impaired subjects. Relatives may request hospital assistance due to pre-admission functional decline. In this last case, the length of stay in hospital should be lower, because it is determined by functional decline rather than by clinical status.

This study presents some limitations. The relatively small sample size does not permit generalization of results. We did not collect data on functional decline after hospital discharge, nor did we collect information on re- habilitation treatments which may have influenced the functional trajectory of elderly patients. In addition, functional impairment two weeks before hospitalization depended on reports from caregivers, and this may represent bias.

Maintenance of functional autonomy is an essential concept in geriatric and rehabilitative medicine. Loss of functional autonomy leads to progressive dependency, which evolves into a lowering of quality of life and loss of dignity. All elderly patients should be considered at risk of functional decline during hospitalization. For all these reasons, long in-hospital stays should be avoided, and patients should be discharged as soon as there is no need for further in-hospital treatment.

Identifying patients at risk of functional decline should be the "gold standard" in assessing the degree of risk, to determine risk factors and to prevent functional decline by developing a model in which, stepwise, all risk factors are prevented by interventions (16).

In conclusion, functional decline two weeks before hospitalization may influence hospital discharge and functional outcomes. This knowledge would be helpful during clinical practice, although we do not provide data on the possible results of dedicated early rehabilitation programs for elderly patients (during the first two days of hospitalization). Future researches should identify well-structured programs involving geriatricians, physiatrists and physiotherapists for improved prognoses in patients with and without functional decline in the weeks immediately before hospitalization.

\section{REFERENCES}

1. Formiga F, Vidaller A, Salazar A, Pujol R. Functional decline in nonagenarians after a visit to an ED. Am J Emerg Med 2003; 21: 509.

2. Cornette P, Swine C, Malhomme B, Gillet J-B, Meert P, D'Hoore $\mathrm{W}$. Early evaluation of the risk of functional decline following hospitalisation of older patients: development of a predictive tool. Eur J Public Health 2005; 16: 203-8.

3. Salvi F, Giorgi R, Grilli A et al. Mini Nutritional Assessment (short form) and functional decline in older patients admitted to an acute medical ward. Aging Clin Exp Res 2008; 20: 322-8.

4. Zanocchi M, Maero B, Francisetti F et al. Multidimensional assessment and risk factors for prolonged hospitalization in the elderly. Aging Clin Exp Res 2003; 15: 305-9.

5. Palmer RM. Acute hospital care of the elderly: minimizing the risk of functional decline. Cleve Clin J Med 1995; 62: 117-28.

6. Kamper AM, Stott DJ, Hyland M, Murray HM, Ford I. Predictors of functional decline in elderly people with vascular risk factors or disease. Age Ageing 2005; 34: 450-5.

7. Knaus WA, Draper EA, Wagner DP, Zimmerman JE. APACHE II: a severity disease classification system. Crit Care Med 1985; 13: 818-29.

8. Katz S, Ford AB, Moskowitz RW, Jackson BA, Jaffe MW. Studies of illness in the aged. The index of ADL: a standardized measure of biological and psychosocial function. JAMA 1963; 185: 914-9. 
9. Lawton MP, Brody EM. Assessment of older people: self-maintaining and instrumental activities of daily living. Gerontologist 1969; 9: 179-86.

10. Folstein MF, Folstein SE, McHugh PR. "Mini Mental State". A practical method for grading the cognitive state of patients for the clinician. J Psychiatr Res 1975; 12: 189-98.

11. Yesavage JA, Brink TL, Rose TL et al. Development and validation of a geriatric depression screening scale: a preliminary report. J Psychiatr Res 1982; 17: 37-49.

12. Gertman PM, Restuccia JD. The Appropriateness Evaluation Protocol: a technique for assessing unnecessary days of hospital care. Med Care 1981; 19: 855-71.

13. Jónsson PV, Noro A, Finne-Soveri $\mathrm{H}$ et al. Admission profile is predictive of outcome in acute hospital care. Aging Clin Exp Res 2008; 20: 533-9.

14. Hirsch $\mathrm{CH}$, Sommers L, Olsen A, Mullen L, Winograd CH. The natural history of functional morbidity in hospitalized older patients. J Am Geriatr Soc 1990; 38: 1296-303.

15. Sager MA, Rudberg MA, Jalaluddin M et al. Hospital admission risk profile (HARP): identifying older patients at risk for functional decline following acute medical illness and hospitalization. J Am Geriatr Soc 1996; 44: 251-7.

16. Hoogerduijn JG, Schuurmans MJ, Duijnstee MS, de Rooij SE, Grypdonck MF. A systematic review of predictors and screening instruments to identify older hospitalized patients at risk for functional decline. J Clin Nurs 2007; 16: 46-57. 